\title{
Sarcoma gástrico primario: reporte de caso y revisión de la literatura
}

\section{Primary gastric sarcoma: Case report and literature review}

Helena Facundo Navia, MD, ${ }^{1 *}$ Juliana Rendón Hernández, MD, ${ }^{2}$ Jorge Mesa, MD. ${ }^{3}$

\footnotetext{
Especialista en Cirugía Gastrointestinal y Endoscopia Digestiva. Instituto Nacional de Cancerología. Bogotá, Colombia.

2 Especialista en Cirugía Gastrointestinal y Endoscopia Digestiva. Instituto Nacional de Cancerología. Bogotá, Colombia.

3 Especialista en Patología Oncológica. Instituto Nacional de Cancerología. Bogotá, Colombia.

*Correspondencia: Helena Facundo Navia, MD, helefacus@gmail.com

Fecha recibido: $22 / 10 / 18$ Fecha aceptado: 18/12/18
}

\begin{abstract}
Resumen
El cáncer gástrico, patología neoplásica de innegable importancia, corresponde en el $90 \%$ de los casos a un adenocarcinoma. Dentro del $10 \%$ restante, los linfomas y los tumores estromales gastrointestinales (Gastrointestinal Stromal Tumor, GIST) constituyen la mayoría. Sin embargo, los sarcomas no GIST siguen siendo un diagnóstico diferencial posible para tener en cuenta y configuran una patología neoplásica de tratamiento fundamentalmente quirúrgico.

En particular, el leiomiosarcoma representa menos del $1 \%$ de los tumores malignos del estómago y la literatura disponible al respecto consiste en reportes de caso o serie de casos. Por su rareza, presentamos este caso clínico y revisamos la literatura relacionada.
\end{abstract}

\section{Palabras clave \\ Leiomiosarcoma, neoplasias gástricas, tumores mesenquimales.}

\begin{abstract} this clinical case and review the literature.

\section{Keywords}

Leiomyosarcoma, gastric neoplasms, mesenchymal tumors.
\end{abstract}

Gastric cancer, a neoplastic pathology of undeniable importance, accounts for $90 \%$ of cases to adenocarcinoma. GIST lymphomas and gastrointestinal stromal tumors are the majority of the other $10 \%$. However, nonGIST sarcomas remain a possible differential diagnosis to keep in mind and constitute a neoplastic pathology whose treatment is fundamentally surgical. Leiomyosarcoma represents less than $1 \%$ of malignant stomach tumors, and the available literature consists of case reports or case series. Because of its rarity, we present

\section{INTRODUCCIÓN}

El leiomiosarcoma gástrico primario es un tumor maligno no epitelial, proveniente de las células del estroma muscular, que frecuentemente metastatiza al hígado y al pulmón. Este es el más común de los sarcomas del estómago, los cuales en todo el mundo constituyen menos del $2 \%$ de los cánceres gástricos (1). Su presentación clínica es sutilmente diferente a la del adenocarcinoma y el diagnóstico prequirúrgico puede ser difícil.
En un artículo clásico de 1948, publicado en JAMA, los doctores Bassler y Peters (2) mencionaron las diferencias clínicas entre los sarcomas y los carcinomas gástricos e invitaron a considerar la posibilidad diagnóstica del sarcoma en el escenario clínico de la neoplasia gástrica maligna.

Entre las características relevantes de los sarcomas están la edad de presentación temprana (en promedio 38 años), la mayor frecuencia en hombres, los cuadros clínicos prolongados en el tiempo con menos caquexia que en el adenocarcinoma y el dolor intenso como síntoma cardinal que 
se asocia con un sangrado masivo, especialmente en estadios avanzados.

Desde aquella publicación, nuestro conocimiento sobre los sarcomas se ha expandido y su clasificación ha sido revisada en diversas ocasiones. Sin embargo, estas observaciones siguen siendo válidas y útiles en la clínica.

Presentamos el caso de un hombre joven con historia familiar de adenocarcinoma gástrico, que debuta con dolor y sangrado digestivo, en quien se confirma el diagnóstico de leiomiosarcoma gástrico primario, y revisamos la literatura.

\section{PRESENTACIÓN DEL CASO}

Se trata de un hombre de 42 años con un cuadro de 3 meses de evolución de dolor abdominal y melenas. Además, presenta antecedentes familiares del abuelo y el padre, quienes tuvieron un adenocarcinoma gástrico a los 70 y los 53 años, respectivamente. La endoscopia alta describe una masa subcardial en la curvatura mayor, vegetante y ulcerada, de $5 \mathrm{~cm}$ de diámetro. La biopsia reporta un pólipo hiperplásico negativo para malignidad.

Al llegar al Instituto Nacional de Cancerología, se realiza una nueva endoscopia y una biopsia, cuyos hallazgos son una lesión polipoide ulcerada, subcardial, de $6 \times 8 \mathrm{~cm}$ con base de implantación de $3 \mathrm{~cm}$, lobulada y firme. La patología corresponde a una lesión mesenquimal fusocelular que, tras el estudio con inmunohistoquímica, es clasificado como un leiomioma. Entre tanto, el paciente presenta dos nuevos episodios de sangrado digestivo que requieren transfusión de hemoderivados y persiste con dolor abdominal de difícil manejo.

La tomografía contrastada de tórax y abdomen evidencia una masa gástrica y se descartan lesiones metastásicas (Figura 1). Se realiza entonces la resección quirúrgica mediante una gastrectomía total con disección ganglionar D1 y la reconstrucción en Y de Roux. La evolución posoperatoria es favorable y es dado de alta a los 7 días.

El diagnóstico patológico definitivo es un leiomiosarcoma de alto grado, de $14 \times 12 \mathrm{~cm}$, con 50 mitosis en 50 campos de alto poder, un Ki67 del $40 \%$, bordes libres y 15 ganglios libres de tumor (Figuras 2 y 3 ). El paciente fue valorado por el servicio de oncología clínica que no indicó una terapia adyuvante. El seguimiento a 28 meses no ha mostrado recidiva tumoral y la condición general del individuo es buena. El dolor y el sangrado digestivo remitieron completamente tras la cirugía.

\section{DISCUSIÓN}

Los sarcomas constituyen el 1-2 \% de los tumores malignos gastrointestinales; la mayoría corresponden a leiomiosarcomas y la localización más frecuente es el estómago $(1,3)$. Otros tipos de sarcomas en el tracto gastrointestinal, como el sinovial, el granulocítico y el sarcoma de Ewing, han sido reportados de manera aislada y representan verdaderas rarezas (4-9).
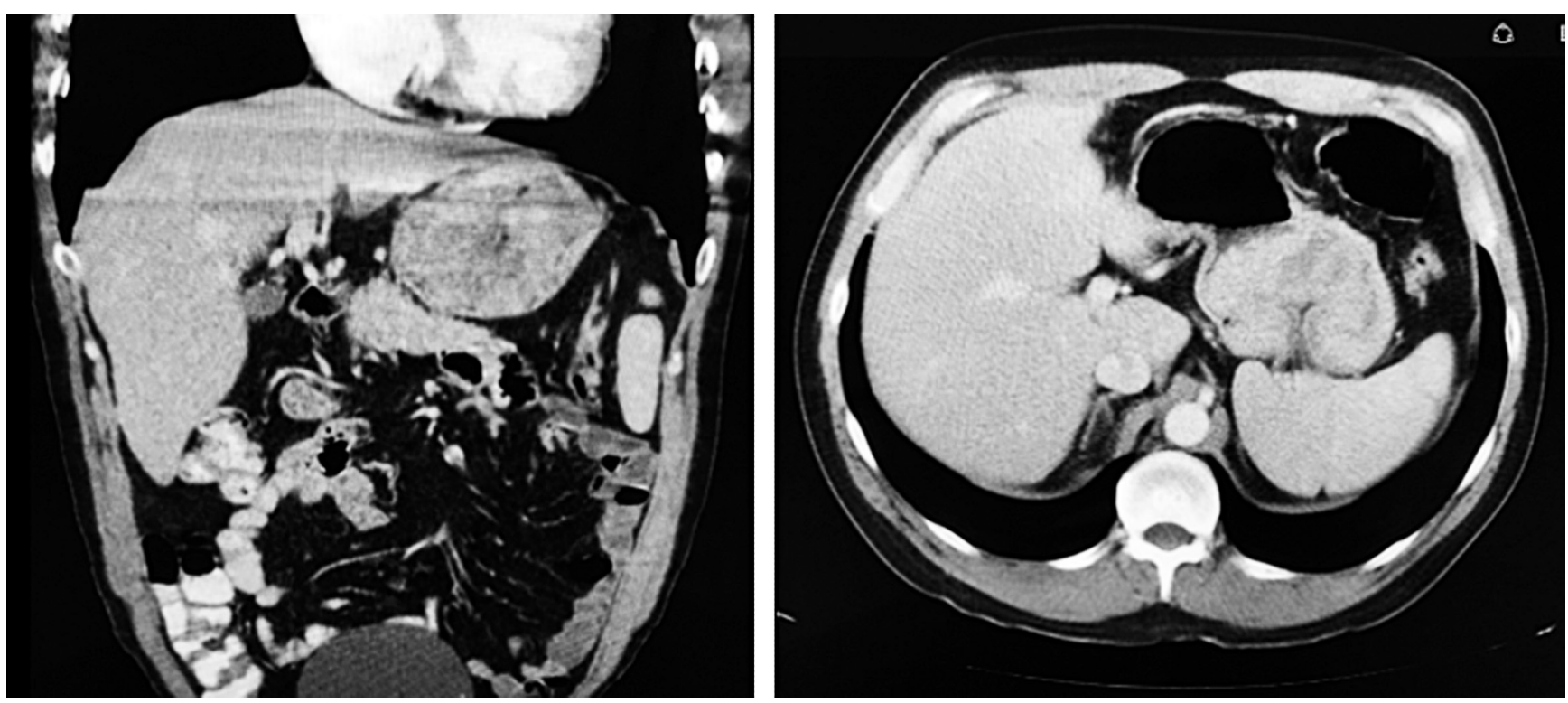

Figura 1. Tomografía axial computarizada (TAC) abdominal que evidencia la masa gástrica. Cortes coronal y axial. 


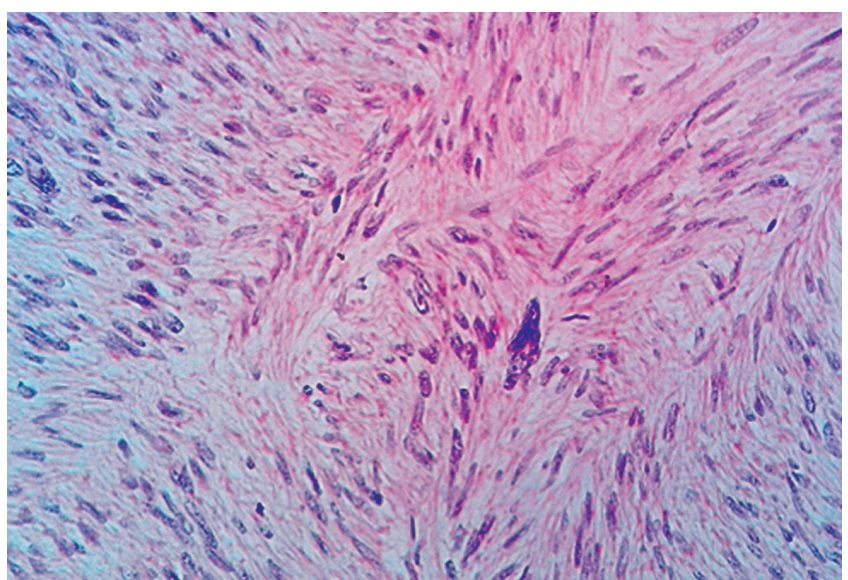

Figura 2. Tinción de hematoxilina-eosina. Células fusiformes con núcleo atípico.

La actividad mitótica ha mostrado ser el indicador aislado más importante en el pronóstico de la enfermedad y la cirugía continúa siendo el pilar del tratamiento (8-12).
Asimismo, el alto grado tumoral, la presencia de metástasis al momento del diagnóstico, el tamaño tumoral y la resección quirúrgica incompleta son factores independientes de pobre pronóstico $(13,14)$.

La naturaleza de estas neoplasias y las particularidades de su comportamiento biológico hacen que la resección quirúrgica en bloque, incluso con órganos vecinos si están comprometidos, tenga importancia capital en disminuir la posibilidad de recidiva. A diferencia de los adenocarcinomas, los sarcomas crecen localmente e invaden por contigüidad, con una baja tendencia a las metástasis ganglionares (15). Sin embargo, la extensión de la disección ganglionar indicada es motivo de discusión, pues existen algunos reportes de compromiso ganglionar hasta en el $15 \%$ de los casos, según la serie (16). Las terapias adyuvantes, como la quimio y la radioterapia, tienen un papel marginal y se aplican principalmente en casos de paliación por lesiones primarias irresecables o recidivas (17).

En la revisión de la clasificación de los sarcomas, el lugar de los leiomiosarcomas ha sido relativamente estable y estos
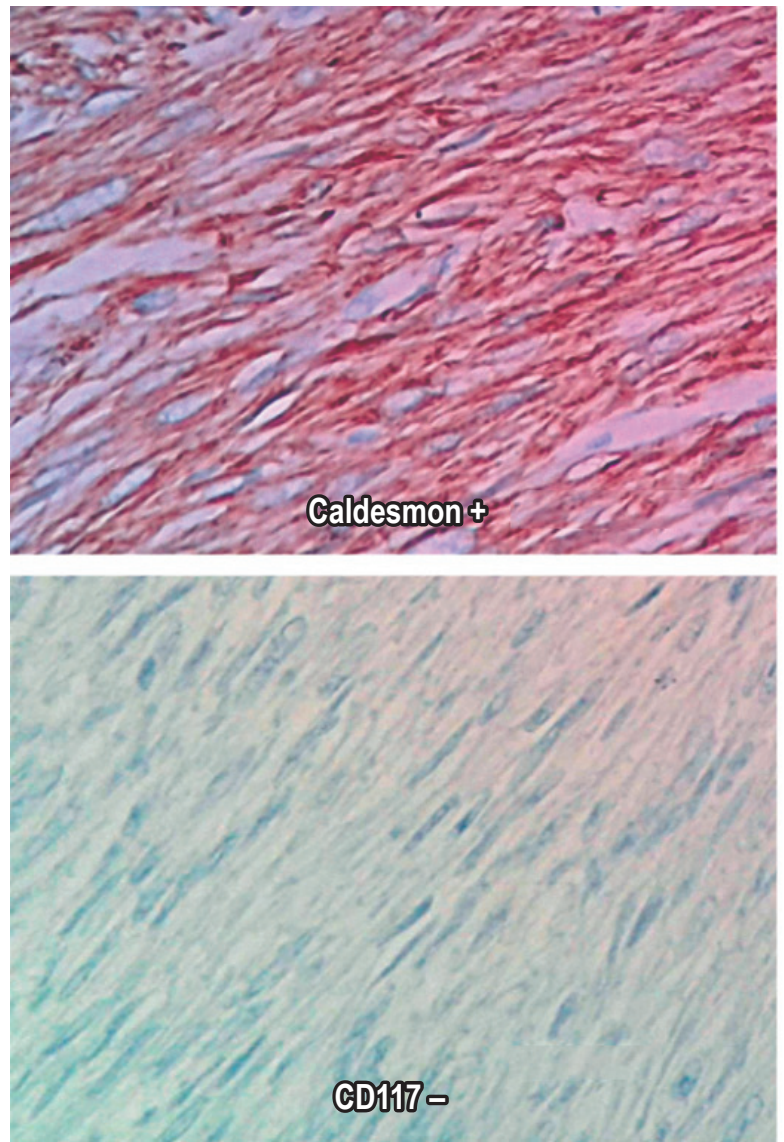
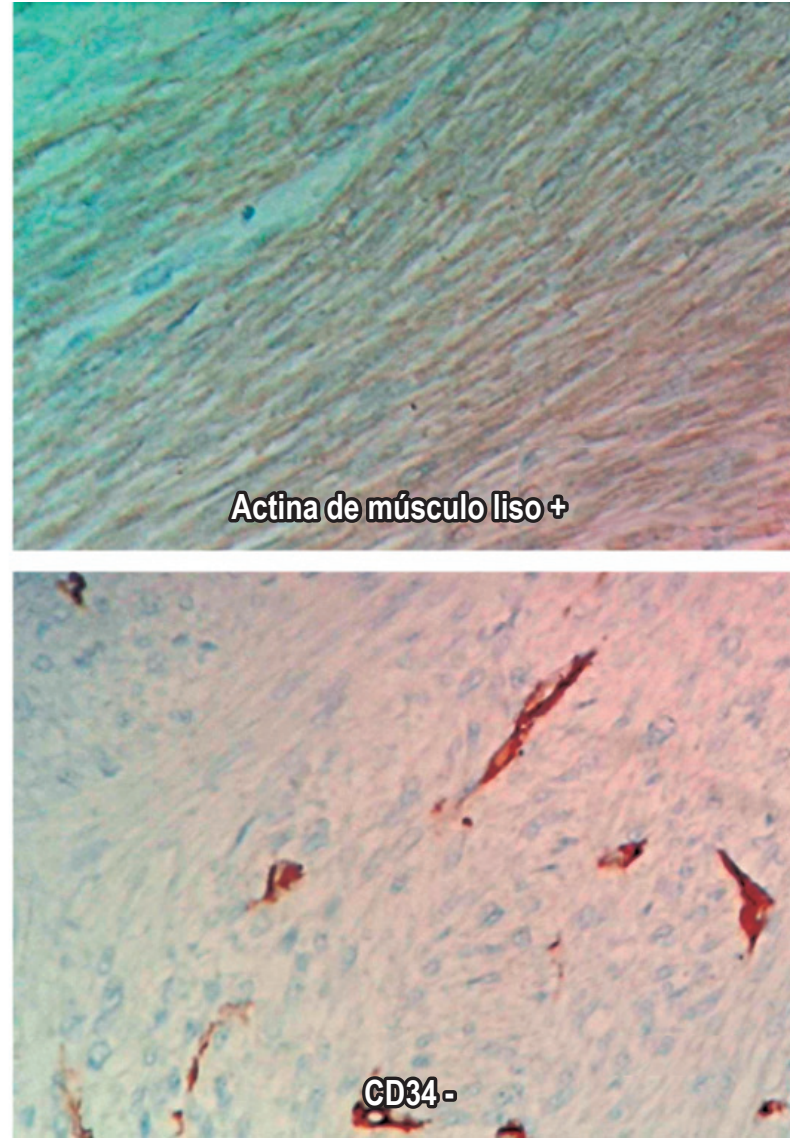

Figura 3. Inmunohistoquímica positiva para actina del músculo liso y caldesmona, negativo para CD117 y CD34. 
se diferencian fundamentalmente de los GIST por las técnicas de inmunohistoquímica (18). Como constituyen lesiones intramurales y extramucosas, el diagnóstico mediante endoscopia y biopsia no es siempre fácil. La sospecha clínica, que lleva a la realización de biopsias en profundidad, y las técnicas de imagen son claves en el establecimiento del diagnóstico prequirúrgico (19). La diferenciación entre el leiomioma y el leiomiosarcoma también es difícil de determinar en las biopsias y a menudo se requiere la pieza quirúrgica a fin de establecer el diagnóstico de certeza, pues la presencia de atipia celular y las mitosis son las claves del diagnóstico diferencial (20).

En la literatura se encuentran múltiples clasificaciones para los sarcomas en general y específicamente para los sarcomas de tejidos blandos. La Federación Nacional de Centros de Lucha contra el Cáncer (FNCLCC) ha propuesto un sistema de clasificación para los sarcomas viscerales y de tejidos blandos, basado en tres aspectos: el grado de diferenciación, la ulceración y la tasa mitótica. Este sistema ha sido validado y es ampliamente utilizado $(21,22)$.

El tamaño tumoral ( $5 \mathrm{~cm}$ es un punto de inflexión), así como la presencia de metástasis al momento del diagnóstico, tienen importancia en el pronóstico y están incluidos en el sistema de estadificación TNM del Comité Conjunto Norteamericano sobre el Cáncer (American Joint Committee on Cancer, AJCC). La supervivencia global a 5 años para los sarcomas gástricos es variable y ha sido reportada entre el 16 y el $56 \%$ según el grado tumoral y el éxito en la resección quirúrgica completa. Las recidivas suelen presentarse en torno a los 2 años de la resección y oscilan entre el 36 y el $60 \%$ (17).

El presente caso es un leiomiosarcoma de alto grado, según la clasificación de la FNCLCC, con un puntaje total de 7 , que corresponde a diferenciación ( 2 puntos), mitosis (3 puntos) y necrosis ( 2 puntos) (Tabla 1$)$.

No existe unanimidad de criterio sobre la necesidad de una disección ganglionar formal, y en general, se sabe de la baja tendencia de esta neoplasia a las metástasis por vía linfática. En contraste, su preferencia por la vía hematógena es reconocida. Prima entonces la decisión del cirujano, basada en los hallazgos clínicos y quirúrgicos. En este caso, no había evidencia macroscópica de adenopatías y se realizó un vaciamiento de primer nivel, D1. Sin embargo, se obtuvieron 15 ganglios, todos negativos para tumor.

Un aspecto particular del caso reportado es la presencia de un adenocarcinoma gástrico en dos familiares del paciente, en primer grado de consanguinidad. Se han descrito reportes en la literatura de la coexistencia entre la histología del adenocarcinoma y los GIST, o del adenocarcinoma y los linfomas gástricos en un mismo paciente (23, 24). No obstante, la asociación familiar entre el sarcoma y el adenocarcinoma gástricos no está referida en los síndromes conocidos de cáncer gástrico heredofamiliar y los autores no conocemos de registros previos con esta característica. Este es un elemento que debe considerarse para la observación clínica y los futuros estudios.

Tabla 1. Parámetros de clasificación de sarcomas de la FNCLCC.

\begin{tabular}{|c|c|}
\hline Aspecto & Criterio \\
\hline \multicolumn{2}{|c|}{ Diferenciación } \\
\hline 1 punto & $\begin{array}{l}\text { Bien diferenciado, estructura que recuerda al tejido } \\
\text { maduro. }\end{array}$ \\
\hline 2 puntos & Moderadamente diferenciado; tipo histológico definido. \\
\hline 3 puntos & $\begin{array}{l}\text { Indiferenciado, estructura que recuerda el tejido } \\
\text { embrionario. }\end{array}$ \\
\hline \multicolumn{2}{|c|}{ Recuento de mitosis } \\
\hline 1 punto & 0-9 en 10 campos de alto poder. \\
\hline 2 Puntos & 10-19 en 10 campos de alto poder. \\
\hline 3 Puntos & $>20$ en 10 campos de alto poder. \\
\hline \multicolumn{2}{|c|}{ Necrosis tumoral (microscópica) } \\
\hline 1 Punto & Sin necrosis. \\
\hline 2 Puntos & $\leq 50 \%$ necrosis. \\
\hline 3 puntos & $>50 \%$ necrosis. \\
\hline \multicolumn{2}{|c|}{ Grado histológico } \\
\hline Grado 1 & Puntaje total: 2-3. \\
\hline Grado 2 & Puntaje total: 4-5. \\
\hline Grado 3 & Puntaje total: 6-8. \\
\hline
\end{tabular}

Modificada de la referencia 21

\section{CONCLUSIONES}

El leiomiosarcoma gástrico primario es una entidad poco frecuente pero claramente establecida, cuyo tratamiento se basa en la resección quirúrgica radical. Su pronóstico es superior al del adenocarcinoma gástrico. Sus características clínicas permiten sospecharlo, pero el diagnóstico de certeza es difícil en la fase prequirúrgica y está basado en los hallazgos de la histopatología, con especial énfasis en la inmunohistoquímica.

\section{REFERENCIAS}

1. McGrath PC, Neifeld JP, Lawrence W, Kay S, Horsley JS, Parker GA. Gastrointestinal sarcomas. Analysis of prognostic factors. Ann Surg. 1987;206(6):706-10. https://doi. org/10.1097/00000658-198712000-00004

2. Bassler A, Peters AG. Distinctions between gastric sarcoma and carcinoma with special reference to the infiltrating types of sarcoma. J Am Med Assoc. 1948;138(7):489-94. https:// doi.org/10.1001/jama.1948.02900070021005

3. Peñaherrera V, Abarca J, Carrillo L. Leiomiosarcoma: reporte de un caso clínico. Rev Med. 2003;9(4):327-330.

4. Billings SD, Meisner LF, Cummings OW, Tejada E. Synovial sarcoma of the upper digestive tract: a report of two cases 
with demonstration of the $\mathrm{X} ; 18$ translocation by fluorescence in situ hybridization. Mod Pathol. 2000;13(1):68-76. https://doi.org/10.1038/modpathol.3880011

5. Zhou W, Vasquez JC, O’Donnell MR, Paz BI. Clinical manifestations of gastrointestinal granulocytic sarcoma requiring surgical treatment. Am Surg. 2001;67(8):764-6.

6. Agaimy A, Gaumann A, SchroederJ, DietmaierW, Hartmann A, Hofstaedter F, et al. Primary and metastatic high-grade pleomorphic sarcoma/malignant fibrous histiocytoma of the gastrointestinal tract: an approach to the differential diagnosis in a series of five cases with emphasis on myofibroblastic differentiation. Virchows Arch. 2007;451(5):949-57. https://doi.org/10.1007/s00428-007-0495-3

7. Kim HS, Kim S, Min YD, Kee KH, Hong R. Ewing's Sarcoma of the Stomach; Rare Case of Ewing's Sarcoma and Suggestion of New Treatment Strategy. J Gastric Cancer. 2012;12(4):258-261. http://dx.doi.org/10.5230/ jgc.2012.12.4.258

8. Dougherty MJ, Compton C, Talbert M, Wood WC. Sarcomas of the gastrointestinal tract. Separation into favorable and unfavorable prognostic groups by mitotic count. Ann Surg. 1991;214(5):569-574. https://doi. org/10.1097/00000658-199111000-00006

9. Weledji EP, Enoworock G, Ngowe MN. Gastric leiomyosarcoma as a rare cause of gastric outlet obstruction and perforation: a case report. BMC Res Notes. 2014;7:479. https:// doi.org/10.1186/1756-0500-7-479

10. Carson W, Karakousis C, Douglass H, Rao U, Palmer ML. Results of aggressive treatment of gastric sarcoma. Ann Surg Oncol. 1994;1(3):244-51. https://doi.org/10.1007/ BF02303530

11. Lehnert T, Sinn HP, Wolf O. Diagnosis and surgical treatment of gastric sarcoma. Onkologie 1994;17:391-396. https://doi.org/10.1159/000218444

12. Koga H, Ochiai A, Nakanishi Y, Sasako M, Mizuno S, Kinoshita T, et al. Reevaluation of prognostic factors in gastric leiomyosarcoma. Am J Gastroenterol. 1995;90(8):1307-12.

13. Medina-Franco H, Eltoum IE, Urist MM, Heslin MJ. Primary gastrointestinal sarcomas. Am Surg. 2000;66(12):1171-5.

14. Farrugia G, Kim CH, Grant CS, Zinsmeister AR. Leiomyosarcoma of the stomach: determinants of longterm survival. Mayo Clin Proc. 1992;67(6):533-6. https:// doi.org/10.1016/S0025-6196(12)60459-5
15. Yao KA, Talamonti MS, Langella RL, Schindler NM, Rao S, Small W, et al. Primary gastrointestinal sarcomas: analysis of prognostic factors and results of surgical management. Surgery. 2000;128(4):604-12. https://doi.org/10.1067/ msy.2000.108056

16. Álvarez R, Arancibia A, Klaassen R, Gutiérrez G, González $\mathrm{R}$, Molina $\mathrm{H}$, et al. Tumores gástricos de estirpe muscular. Rev Chil Cir. 2003;55(5):470-475.

17. BeyroutiMI,Beyrouti R,Amar M, Frikha F,Abid M,Affes N, et al. Sarcomes gastriques. La Presse Médicale. 2008;37(3):e60e66. https://doi.org/10.1016/j.lpm.2007.05.004

18. Fletcher $C D$. The evolving classification of soft tissue tumours - an update based on the new 2013 WHO classification. Histopathology. 2014;64(1):2-11. https://doi. org/10.1111/his.12267

19. ESMO/European Sarcoma Network Working Group. Soft tissue and visceral sarcomas: ESMO Clinical Practice Guidelines for diagnosis, treatment and followup. Ann Oncol. 2014;25 Suppl 3:iii102-12. https://doi. org/10.1093/annonc/mdu254

20. Miettinen M. Smooth muscle tumors of soft tissue and non-uterine viscera: biology and prognosis. Mod Pathol. 2014;27 Suppl 1:S17-29. https://doi.org/10.1038/modpathol.2013.178

21. Baig MA. Evaluation of performance of various histological grading systems of soft tissue sarcomas and the prognosis (metastatic risk and survival rate). Int J Res Med Sci. 2015;3(9):2394-2401. https://doi.org/10.18203/23206012.ijrms20150637

22. Guillou L, Coindre JM, Bonichon F, Nguyen BB, Terrier P, Collin F, et al. Comparative study of the National Cancer Institute and French Federation of Cancer Centers Sarcoma Group grading systems in a population of 410 adult patients with soft tissue sarcoma. J Clin Oncol. 1997;15(1):350-62. https://doi.org/10.1200/JCO.1997.15.1.350

23. Khoshnevis J, Rakhshan A, Sobhiyeh MR, Gholizadeh B, Rahbari A, Adhami F, et al. Simultaneous gastric adenocarcinoma and gastrointestinal stromal tumor of the stomach: a case report. Iran J Cancer Prev. 2013;6(1):55-58.

24. Weng CH, Wang RC, Wu CC, Lin TH. Treatment of synchronous adenocarcinoma and lymphoma of the stomach: A case report. Mol Clin Oncol. 2016;5(6):783-785. https:// doi.org/10.3892/mco.2016.1044 